\title{
The International Depression Epidemiological Study (TIDES): unfinished business?
}

\author{
A Kevin Webb, ${ }^{1}$ Mandy Bryon ${ }^{2}$
}

The remarkable improvement in survival for patients with cystic fibrosis (CF) is one of the great success stories of modern clinical medicine. Over a period of three decades, a disease associated with childhood dying (as parents of CF children were informed) has become one of adulthood survival into the fifth and sixth decades of life. CF is the most complex of diseases. It remains (despite recent advances) a chronic multisystem incurable disease in a survivor population who are beginning to lead normal working and domestic lives. This improved survival is a direct result of holistic care delivered by multidisciplinary teams (MDT) based in paediatric and adult CF centres. ${ }^{1}$ The establishment of CF centres around the world has enabled large evidence-based clinical trials, patient registries, best practice guidelines and thereby patient-centred care.

Although CF patients appear to cope remarkably well with a disease which places a huge burden of care on an often busy working and social life, it is not surprising as detailed in several small series that CF patients suffer to varying degrees from heightened anxiety and depression. $^{2-4}$ There is a non-negotiable requirement of CF adults and children to provide lifelong self-care to maintain disease stability and to make the most of a normal quality of life. Perceived poor quality of life can result in a despondency with treatment and poor adherence to the daily regimen leads quickly to deterioration of health. ${ }^{5}$ Lifelong medical conditions are not managed by medicine alone, there is a psychological cost and this paper is commended for having elevated the issue of potential psychological impairment in CF. The provision of expert psychosocial care is an essential component of the holistic care provided by CF centres. However, there is no real consensus in published studies about the level of anxiety and depression in CF patients; probably due

\footnotetext{
${ }^{1}$ Manchester Adult CF Centre, University Hospitals, Manchester, UK; ${ }^{2}$ Great Ormond St Hospital for Children NHS Trust, London, UK
}

Correspondence to Mandy Bryon, Great Ormond St Hospital for Children NHS Trust, London WC1N 3JH, UK; Mandy.bryon@gosh.nhs.uk to small numbers of CF patients and the use of different questionnaires to measure different psychological variables.

Therefore, the publication of this article (The International Depression Epidemiological Study (TIDES)) which has studied anxiety and depression in $154 \mathrm{CF}$ centres in nine countries in a large number of $\mathrm{CF}$ adults, children and parent carers is timely. ${ }^{7}$ Does this article break new ground and so provide a validated roadmap for assessing levels of anxiety and depression in CF centres across the world? At face value, the conclusions to the study are straightforward: namely, there are significant elevations of anxiety and depression symptoms in all groups across all countries and these symptoms should be addressed annually in a systematic way. An international guidelines committee has been created as a result of the study to formulate recommendations leading to a change in medical practice. However, the devil lies in the detail of the methods used and several inconsistencies in the reporting. There are a large number of participants, though errors are made in reporting just how many, the abstract reports 6088 though within the results they add up to 6025 . It is really not clear what the rates of depression are, there are inconsistencies between text and tables, what is the rate of depressive symptomatology in say adolescents? Is it 5\%, 19\%, 10\% (table $1[7]$ ) or $11 \%$ or $17 \%$ (table 2[7]).

Confusion arises when two screening tools were used: the Hospital Anxiety and Depression Scale (HADS) which has been used widely in Europe but not in the USA and the Center for Epidemiological Studies Depression-Scale (CES-D) which measures depression but not anxiety. Not all the nine countries involved in the TIDES used both measures; this calls into question the methodology. Indeed in the discussion, the authors appear to regret having used the HADS measure citing lack of sensitivity, underestimation of symptoms and state it should not be recommended for screening. ${ }^{8-10}$ Does this mean the authors are suggesting we disregard the HADS data? Therefore, four of the nine participating countries report unreliable data and would reduce the number of participants by 3313 . It is unclear why the authors go to such great extent to dismiss the HADS measure when more than half of the data included in this study are collected from that questionnaire. Why so critical of one of their own selected measures, is it maybe because the HADS reported low rates of depressive symptomatology? The HADS is the only measure included in the study that also measured anxiety. What is the reader to make of the reports on anxiety? Is the HADS also lacking sensitivity in the screening of anxiety? Two questions that immediately need to be asked therefore are that if all the subjects did not use both questionnaires which of the two symptoms is more prevalent in terms of anxiety and depression and which is the more important in terms of a therapeutic approach?

Of interest, a recent publication (with three of the same authors) considered anxiety and depression in $2065 \mathrm{CF}$ patients from 39 centres in the UK. The UK data used were from the original TIDES and employed the HADS measure. It concluded anxiety and depression in CF patients were similar to the general population. ${ }^{11}$ Allowing that this is a subset from the main study, the numbers of CF patients who participated are significantly large (the largest sample included) and raises concerns as to whether the authors common to both papers were in agreement as to the conclusions to their studies!

Despite the paper reporting many varying rates of depression and anxiety symptomatology in the tables and Results section, the discussion tells us that elevated rates of depression were reported at $17 \%$ across countries and regardless of which screening tool was used. This is not acceptable. The authors decry the HADS, yet feel it is fine to pool the HADS and CES-D data to produce a figure of $17 \%$, irrespective of the statistical error made in pooling the data from two measures which are not the same; it is disingenuous to dismiss one measure as unreliable but to include the scores in final figures. As an epidemiological study, the paper neither defends the sampling methods adequately nor are the methods for controlling for variability across countries explained sufficiently. The reader, therefore, does not have confidence that variations in $\mathrm{CF}$ parameters are standardised across the whole sample. Additionally, the authors cannot state depressive symptomatology is found at a rate two times that reported in community samples because that statistical analysis was not done in this study.

The CES-D and the HADS are screening measures; they are not diagnostic. 
They are intended for use in individuals considered by a range of indicators to be at risk for mood disturbance. ${ }^{12}$ Most commonly, they are administered as part of a comprehensive assessment by health professionals to help direct appropriate intervention. The authors ignore this and repeatedly state their study revealed high rates of depression and anxiety. In fact, the study revealed variable rates of symptomatology gained from screening tools, one of which is now dismissed.

There is overlap with some of the authors and an already established international committee to make recommendations for annual screening of depression and anxiety. The authors of this paper have pre-empted the committee's recommendations by concluding that as a result of their study they suggest using two completely different questionnaires the PHQ-9 which measures only symptoms of depression and the GAD-7 which measures only symptoms of anxiety. This ultimately undermines the findings of this study; if neither the HADS nor now the CES-D is a reliable screening tool, confidence is not inspired in the reader that the TIDES has delivered on its promise. We still don't know the prevalence of depression and anxiety in people with CF and their carers because the wrong measures were used.

The paper concludes that their findings indicate that a psychosocial annual review of patients is essential as part of standard care for people with CF. It may be that the results of this study were such a long time in coming; they have been superseded by international recognition of the mental health needs of people with $\mathrm{CF}$ and their families. In fact, many countries now have clinical psychologists and social workers as standard core provision in the MDT. The European Standards of Care report this in detail. ${ }^{13}$

Contributors Both MB and AKW contributed to this manuscript.

Competing interests None.

Provenance and peer review Commissioned; internally peer reviewed.

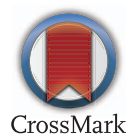

To cite Webb AK, Bryon M. Thorax 2014;69:10671068.

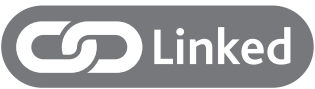

- http://dx.doi.org/10.1136/thoraxjnl-2014-205983

Thorax 2014;69:1067-1068.

doi:10.1136/thoraxjnl-2014-206200

\section{REFERENCES}

1 Mahadeva R, Webb K, Westerbeck C, et al. Clinical outcomes in relation to care in centres specialising in cystic fibrosis: cross sectional study. BMJ 1998;316:1771-5.

2 Yohannes AM, Willgoss TG, Fatoye FA, et al. Relationship between anxiety, depression, and quality of life in adult patients with cystic fibrosis. Respir Care 2012;57:550-6.
3 Modi AC, Driscoll KA, Montag-Lieffling K, et al. Screening for symptoms of depression and anxiety in adolescents and young adults with cystic fibrosis. Pediatr Pulmonol 2011;46:153-9.

4 Latchford G, Duff AJA. Assessing anxiety, depression and suicidal ideation in a single CF centre. J Cyst Fibros 2013;12:794-6.

5 Gee L, Abbott J, Hart A, et al. Associations between clinical variables and quality of life in adults with cystic fibrosis. J Cystic Fibros 2005;4:59-66.

6 Herrmann C. International experiences with the Hospital and Anxiety and Depression Scale-a review of validation and clinical results. I Psychosom Res 1997;42:17-41.

7 Quittner AL, Goldbeck L, Abbott J, et al. Prevalence of depression and anxiety in patients with cystic fibrosis and parent caregivers: results of The International Depression Epidemiological Study across nine countries. Thorax 2014;69:1090-7.

8 Martin CR. What does the Hospital and Anxiety Depression (HADS) really measure in liaison psychiatry settings? Curr Psychiatry Rev 2005:1:69-73.

9 Cameron IM, Crawford JR, Lawton K, et al. Psychometric comparison of PHQ-9 and HADS for measuring depression severity in primary care. $\mathrm{Br} \mathrm{J}$ Gen Pract 2008:58:32-6.

10 Cameron IM, Cardy A, Crawford JR, et al. Measuring depression severity in general practice; discriminatory performance of PHQ-9.HADS-D and BDI-11. Brit J Gen Pract 2011;61:e426.

11 Duff AJ, Abbott J, Cowperthwaite C, et al. Depression and anxiety in adolescents with cystic fibrosis in the UK: a cross sectional study. J Cyst Fibros. Published Online First: 15 Mar 2014. doi:10.1016/j.jcf.2014.02.010

12 Zigmond AS, Snaith RP. The hospital anxiety and depression scale. Acta Psychiatr Scand 1983;67:361-70.

13 Conway S, Balfour-Lynn IM, De Rijcke K, et al. European Cystic Fibrosis Society Standards of Care: framework for the Cystic Fibrosis Centre. J Cyst Fibros 13:S3-22. 